\title{
A PRIORI ESTIMATES FOR CONTINUATION PROBLEMS FOR ELLIPTIC AND PRINCIPALLY NORMAL DIFFERENTIAL EQUATIONS $\left({ }^{1}\right)$
}

\author{
BY \\ PAUL E. SAYLOR $\left({ }^{2}\right)$
}

Introduction. There are well-known procedures due to Douglas [6], [7] for finding an approximation to an analytic or harmonic function in the unit disk when data are given at a finite number of points inside. The success of the error analysis depends on estimates which show that an analytic or harmonic function defined and bounded on the unit disk depends continuously on its restriction to a concentric disk of smaller radius.

In general, the problem of determining a function in a given class from its restriction is called the continuation problem. When the function exists, is unique and depends continuously on its restriction, the continuation problem is said to be well posed in the sense of Hadamard. For practical purposes continuous dependence must be taken to mean Hölder continuous dependence. When there is Hölder continuous dependence, the continuation problem, in the terminology of F. John, is said to be well behaved. The estimates used in the error analysis of Douglas's numerical procedures, due to Hadamard [12] for analytic functions and to Miller [16] for harmonic functions, are an example of this kind. They show that the continuation problem is well behaved for bounded analytic functions and for bounded harmonic functions in the plane.

A more general result is due to F. John, who has shown in [14] that the continuation problem for solutions of linear analytic elliptic equations of arbitrary order in any number of variables is well behaved if a bound on the solutions is prescribed.

The subject of this paper is the nature of the continuation problem for the solutions of nonanalytic elliptic equations in more than two variables. The main result is that the continuation problem is well behaved for bounded solutions of (a class of) elliptic equations with $C_{1}$ coefficients and of arbitrary order. Continuous dependence of the solution on its restriction is expressed in terms of the uniform norm.

This result is derived in $\$ \$ 3$ and 4. The derivation follows from the $L_{2}$ estimates with a weight function that Hörmander used in [13] to prove, among other results,

Received by the editors May 31, 1968.

(1) This work is contained in the author's doctoral thesis written under the direction of Jim Douglas, Jr.

${ }^{(2)}$ Department of Computer Science, University of Illinois. I would like to state here my appreciation to Professor Douglas for his interest and guidance. 
uniqueness for the Cauchy problem. The generality of the estimates also makes it possible to prove that the continuation problem for uniformly bounded solutions of (a class of) principally normal equations is well behaved. Continuous dependence, however, is not expressed in terms of the uniform norm.

The main assumption on which the estimates of Hörmander and the derivation are based is that continuity must be considered only across surfaces that are strongly pseudo-convex with respect to the operator. This assumption is discussed in the last section. In particular, it is quite easy to show that any real-valued $C^{2}$ function with a nonvanishing gradient is strongly pseudo-convex with respect to a second order elliptic operator with real $C^{1}$ coefficients.

The study of the continuation problem for the solutions of nonanalytic elliptic equations in more than two variables begins with a three sphere theorem due to Landis [15]. Landis's theorem shows that the continuation problem for the solutions of second order equations in $n$ variables with real coefficients is well behaved, under additional mild restrictions to be stated.

It seems worth stating Landis's theorem as a convenient reference since the main result can be viewed as a generalization of it. In order to state the theorem, suppose the equation has the form

$$
\sum_{i, k=1}^{n} a_{i k}(x) \frac{\partial^{2} u}{\partial x_{i} \partial x_{k}}+\sum_{i=1}^{n} b_{i}(x) \frac{\partial u}{\partial x_{i}}+c(x) u=0 .
$$

Assume that the coefficients $a_{i k}$ are twice continuously differentiable; that all the coefficients are bounded in absolute value by the constant $M$; that all of the partial derivatives of first and second order of $a_{i k}$ are bounded in absolute value by $M$; that the remaining coefficients are continuously differentiable and their derivatives are bounded by $M$; and finally that $c(x) \leqq 0$.

THEOREM (LANDIS). In a sphere $Q$ of radius $r_{2}<1$ with center at the origin assume there is defined a solution of equation (i), continuous in the closed sphere. Let

$$
M(r)=\max _{|x|=r}|u(x)|
$$

Then for any $r_{1}$ and $r, 0<r_{1}<r<r_{2}$, the following inequality is valid:

$$
\ln M(r) \leqq \ln M\left(r_{1}\right) \frac{\ln c r / r_{2}}{\ln r_{1} / r_{2}}+\ln M\left(r_{2}\right) \frac{\ln c r / r_{1}}{\ln r_{2} / r_{1}}+\ln \ln \frac{c}{r},
$$

where $c$ is a constant depending on the ellipticity constant, on $M$ and on the dimension $n$ of the space.

The result of Landis has recently been extended by Gerasimov in [10]. With a weaker condition on $b_{i}$, Gerasimov has obtained a less explicit version of (ii) for products of operators of the form (i). His method of proof seems to be a development of the method Landis used. 
The idea of Landis's proof is clear, although the details of it are obscure. The idea is to modify the method of Heinz and Cordes used to establish strong unique continuation for the solutions of a class of second order elliptic equations. Note that an elliptic equation is said to possess the strong unique continuation property if every solution which vanishes at a point of infinite order, that is, tends to zero faster than any power of the distance from this point, vanishes identically. Strong unique continuation was established by Carleman [4], Bers [2], Douglis [8] and Morrey [17] for second order equations in the plane. The proof of Carleman uses the method of $L_{1}$ estimates for the solution in terms of the operator with appropriate weight functions. The same idea has been used for all later work on the uniqueness question with the $L_{2}$ norm taking the place of the $L_{1}$ norm for more than two variables. However, the method of Carleman's proof did not generalize to higher dimensions, and uniqueness theorems for second order operators were first obtained by Müller [18], Heinz [11], Cordes [5], and Aronszajn [1]. The paper of Cordes is complicated, for the idea, which Landis applied to obtain a three circle theorem, is to derive $L_{2}$ estimates from a polar form for second order operators that is obtained by a complicated transformation to spherical coördinates. This generalizes the method of Heinz, valid when the principal part is the Laplacian.

A simplified approach to obtaining $L_{2}$ estimates, which can be used to prove a type of strong unique continuation theorem, was given by Protter in [19]. Moreover, it is clear how to modify Protter's derivation to obtain estimates that show that the continuation problem for second order elliptic equations with real $C^{2}$ coefficients is well posed. Unfortunately, the dependence on the restriction is not Hölder continuous. Thus, this approach to the problem does not yield an estimate which is practical. In addition, it does not seem to generalize to higher order equations.

An approach to the continuation problem based on strong unique continuation estimates is somewhat misleading. The whole question of finding estimates to apply to the continuation problem is handled more easily by looking at the estimates used to obtain weak unique continuation rather than strong unique continuation. An equation is said to possess the weak unique continuation property if every solution which vanishes in an open set vanishes identically. When weak unique continuation estimates are applied to the continuation problem, it becomes apparent they are a simplification over strong unique continuation estimates. The simplification is that weak unique continuation estimates do not need to be rederived for solutions that are not zero on an open set, whereas strong unique continuation estimates do need to be modified for solutions without a zero of infinite order.

The connection of the weak unique continuation property for elliptic equations with the $L_{2}$ estimates Hörmander used to prove uniqueness for the Cauchy problem is that the weak unique continuation property and uniqueness for the Cauchy problem are equivalent. 
1. Notation. Let

$$
P(x, D)=\sum_{|\alpha| \leqq m} a_{\alpha}(x) D^{\alpha}
$$

be a partial differential operator, and let $P_{m}(x, D)$ be the principal part. The following notation will be used extensively,

$$
P_{m}^{(j)}(x, \xi)=\partial P_{m}(x, \xi) / \partial \xi_{j}, \quad P_{m, j}(x, \xi)=\partial P_{m}(x, \xi) / \partial x_{j} .
$$

The operator $\bar{P}$ is obtained from $P$ by conjugating the coefficients of $P$.

Also, the set $C^{K}(\bar{\Omega})$ is the set of functions in $\bar{\Omega}$ which can be extended to functions in $C^{K}\left(R_{n}\right)$.

2. Pseudoconvexity and principally normal operators. The concepts defined in this section are due to Hörmander.

Definition A [13, p. 203]. Let $\psi \in C^{2}$ be a real-valued function defined in a neighborhood of a point $x^{0}$ and assume that $\nabla \psi\left(x^{0}\right) \neq 0$. Let $P(x, D)$ be a differential operator of order $m$. The oriented surface defined by the equation $\psi(x)=\psi\left(x^{0}\right)$ will be called pseudo-convex with respect to $P$ at the point $x$ if

$$
\begin{aligned}
\sum_{j, k=1}^{n} \frac{\partial^{2} \psi}{\partial x_{j} \partial x_{k}} P_{m}^{(j)}(x, \xi) \bar{P}_{m}^{(k)}(x, \xi) & \\
& +\operatorname{Re} \sum_{j, k=1}^{n}\left[P_{m, k}^{(j)}(x, \xi) \bar{P}_{m}^{(k)}(x, \xi)-P_{m, k}(x, \xi) \bar{P}_{m}^{(k j)}(x, \xi)\right] \frac{\partial \psi}{\partial x_{j}}>0
\end{aligned}
$$

for all $\xi \neq 0$ in $R_{n}$, satisfying the equations

$$
P_{m}(x, \xi)=0, \quad \sum_{1}^{n} P_{m}^{(j)}(x, \xi) \frac{\partial \psi}{\partial x_{j}}=0 .
$$

The surface is called strongly pseudo-convex with respect to $P$ at the point $x$ if, in addition,

$$
\sum_{j, k=1}^{n} \frac{\partial^{2} \psi}{\partial x_{j} \partial x_{k}} P_{m}^{(j)}(x, \zeta) \overline{P_{m}^{(k)}(x, \zeta)}+\tau^{-1} \operatorname{Im} \sum_{1}^{n} P_{m, k}(x, \zeta) \overline{P_{m}^{(k)}(x, \zeta)}>0
$$

for all $\zeta=\xi+i \tau \operatorname{grad} \psi(x)$, with $\xi \in R_{n}$ and $0 \neq \tau \in R_{1}$, satisfying the equations

$$
P_{m}(x, \zeta)=0, \quad \sum_{1}^{n} P_{m}^{(j)}(x, \zeta) \frac{\partial \psi}{\partial x_{j}}=0
$$

Hörmander, in [13], proved a theorem that gives estimates valid for operators with real coefficients. These estimates also hold for principally normal operators, defined below, provided a more restrictive differentiability condition is imposed on the coefficients. Moreover, operators with real coefficients are principally normal. Thus, a weaker version of the theorem that gives estimates for operators with real coefficients is also valid for principally normal operators. 
Definition B [13, p. 199]. Let

$$
C_{2 m-1}(x, \xi)=2 \operatorname{Im} \sum_{1}^{n} P_{m, j}(x, \xi) \overline{P_{m}^{(j)}(x, \xi)}=0
$$

if $P_{m}(x, \xi)=0, \xi \in R_{n}, x \in \Omega . P(x, D)$ is said to be principally normal in $\bar{\Omega}$ if the coefficients of $P_{m}$ are in $C^{1}(\bar{\Omega})$ and there exists a differential operator $Q_{m-1}(x, D)$, homogeneous of degree $m-1$ in $D$, with coefficients in $C^{1}(\bar{\Omega})$, such that

$$
C_{2 m-1}(x, \xi)=2 \operatorname{Re} P_{m}(x, \xi) \overline{Q_{m-1}(x, \xi)}, \quad \xi \in R_{n} .
$$

3. Derivation of Hölder continuity. Assume the hypotheses of the theorem of Hörmander on Cauchy uniqueness [13, Theorem 8.9.1, p. 224]. Let $P(x, D)$ be a differential operator of order $m$ with bounded, measurable coefficients in a neighborhood $\Omega$ of a point $x^{0}$. Also assume either $P$ is principally normal and that the coefficients in the principal part are in $C^{2}(\Omega)$ or else that $P_{m}$ has real $C^{1}$ coefficients or that $P_{m}$ is elliptic with $C^{1}$ coefficients. Let $\psi$ be a real valued function in $C^{2}(\Omega)$ such that $\operatorname{grad} \psi\left(x^{0}\right) \neq 0$ and the level surface defined by $\psi(x)=\psi\left(x^{0}\right)$ is strongly pseudo-convex at $x^{0}$.

Following Hörmander, let $\psi_{2}$ be the Taylor expansion of $\psi$ of second order at $x^{0}$. By [13, Theorem 8.6.1], there is an open set, $\omega$, about $x^{0}$ in which $\psi_{2}$ has pseudoconvex level surfaces. By the same theorem for $\varepsilon>0$ sufficiently small, $\psi^{\prime}(x)=\psi_{2}(x)$ $-\varepsilon\left|x-x_{0}\right|^{2}$ has pseudo-convex level surfaces in an open set $\omega^{\prime}$ about $x^{0}$. For this $\varepsilon$, there is an open set $\omega^{\prime \prime}$ about $x^{0}$ such that

$$
\left|\psi_{2}(x)-\psi(x)\right|<\frac{1}{2} \varepsilon\left|x-x^{0}\right|^{2}
$$

in $\omega^{\prime \prime}$. It follows that $\psi^{\prime}(x)<\psi(x)$ for $x \in \omega^{\prime \prime}$ and $x \neq x^{0}$. Thus,

$$
\left\{x: \psi^{\prime}(x)=\psi\left(x^{0}\right), x \neq x^{0}, x \in \omega^{\prime \prime}\right\} \subset\left\{x: \psi\left(x^{0}\right)<\psi(x)\right\} .
$$

Assume the closure of $\omega$, the closure of $\omega^{\prime}$, and the closure of $\omega^{\prime \prime}$ are contained in $\Omega$. Let $\Omega^{\prime}=\omega \cap \omega^{\prime} \cap \omega^{\prime \prime}$. Set $\phi=e^{\lambda \psi^{\prime}}$. The $L_{2}$ estimates now hold with weight function $\phi$. More precisely, it follows by [13, Theorems 8.3.1, 8.4.3, 8.5.2, and 8.6.3], for $\lambda$ sufficiently large, that

$$
\sum_{|\alpha| \leqq m} \int\left|D^{\alpha} v\right|^{2} e^{2 \tau \phi} d x \leqq c \tau \int|P(x, D) v|^{2} e^{2 \tau \phi} d x
$$

for $v \in C_{0}^{\infty}\left(\Omega^{\prime}\right)$ if $P$ is elliptic, and

$$
\sum_{|\alpha| \leqq m-1} \tau \int\left|D^{\alpha} v\right|^{2} e^{2 \tau \phi} d x \leqq c \int|P(x, D) v|^{2} e^{2 \tau \phi} d x
$$

for $v \in C_{0}^{\infty}\left(\Omega^{\prime}\right)$ if $P(x, D)$ is principally normal or has real coefficients, or is elliptic.

Observe that by the choice of $\psi^{\prime}$, there is an open set $S \subset \Omega^{\prime}$ such that

$$
S \supset\left\{x: x \in \Omega^{\prime}, \phi(x) \geqq \phi\left(x^{0}\right), x \neq x^{0}\right\}
$$

and

$$
S \subset\left\{x: \psi(x)>\psi\left(x^{0}\right)\right\}
$$




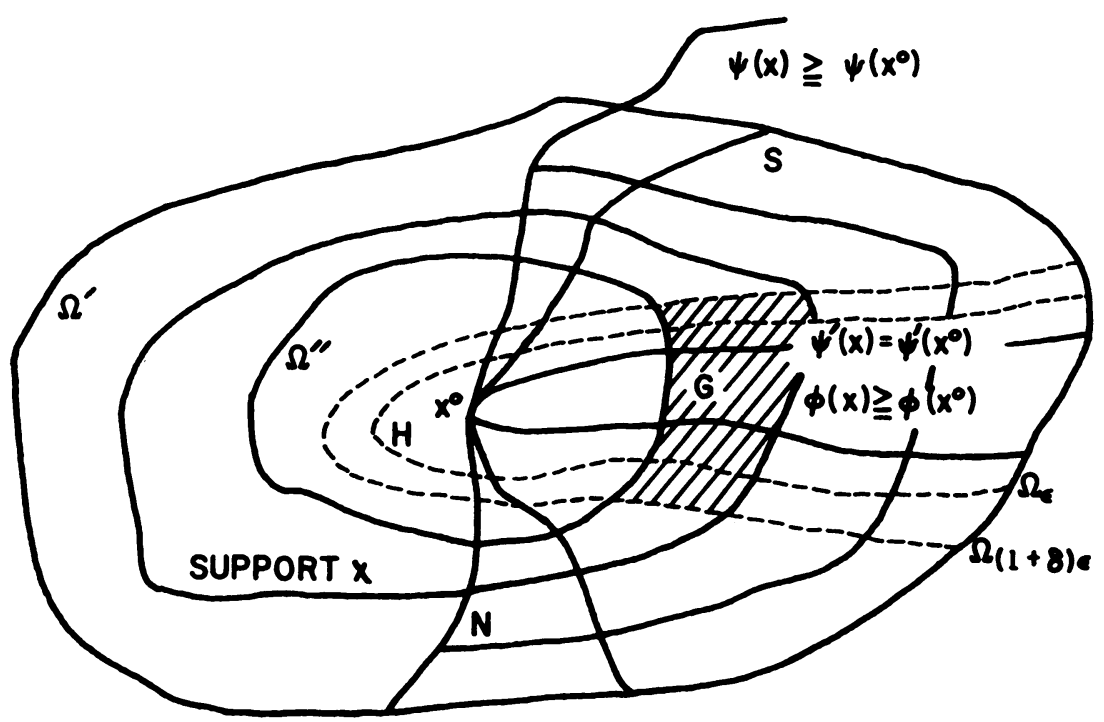

Choose $\chi \in C_{0}^{\infty}\left(\Omega^{\prime}\right)$ to be 1 in a neighborhood $\Omega^{\prime \prime}$ of $x^{0}$. Let

$$
\Omega_{\varepsilon}=\left\{x: x \in \Omega^{\prime}, \phi(x)>\phi\left(x^{0}\right)-\varepsilon\right\}, \quad H=\Omega^{\prime \prime} \cap \Omega_{\varepsilon} \cap\left\{x: \psi(x)<\psi\left(x^{0}\right)\right\},
$$

and, for $\delta>0$,

$$
G=\{x: 0<\chi(x)<1\} \cap \Omega_{(1+\delta) \varepsilon} .
$$

Since the inclusions (6) and (7) hold it is possible to pick $\varepsilon$ and $\delta$ such that $\bar{G} \subset$ $\left\{x: \psi\left(x^{0}\right)<\psi(x)\right\}$.

If $P(x, D) u(x)=0$ in $\Omega$, let $v=\chi u$. Since $v$ may be approximated in $H_{(m)}$ by functions in $C_{0}^{\infty},(4)$ or (5) holds for $v$ according as whether $P$ is elliptic or whether $P$ is principally normal or has real coefficients or is elliptic. Assume $P$ is elliptic in the steps which follow. The case for $P$ not elliptic will be treated later in the section.

Observe that $u(x)=v(x)$ for $x \in H$ and that $P(x, D) v(x)=0$ for $x \in \Omega_{(1+\delta) \varepsilon}$, unless $x \in G$. Also, $\phi\left(x^{0}\right)-\varepsilon<\phi(x)$ for $x \in \Omega_{\varepsilon}$ and $\phi(x) \leqq \phi\left(x^{0}\right)-(1+\delta) \varepsilon$ for

$$
x \in \Omega^{\prime} \backslash \Omega_{(1+\delta) \varepsilon} .
$$

For $\tau$ sufficiently large, say $0<\tau_{0} \leqq \tau$, it follows from (4) that

$$
\begin{aligned}
\sum_{|\alpha| \leqq m} \int_{H}\left|D^{\alpha} u\right|^{2} e^{2 \tau\left[\phi\left(x^{0}\right)-\varepsilon\right]} d x= & \sum_{|\alpha| \leqq m} \int_{H}\left|D^{\alpha} v\right|^{2} e^{2 \tau\left[\phi\left(x^{0}\right)-\varepsilon\right]} d x \\
\leqq & \sum_{|\alpha| \leqq m} \int_{\Omega_{\varepsilon}}\left|D^{\alpha} v\right|^{2} e^{2 \tau\left[\phi\left(x^{0}\right)-\varepsilon\right]} d x \\
\leqq & c \tau \int_{\Omega^{\prime} \mid \Omega_{(1+\delta) \varepsilon}}|P(x, D) v|^{2} e^{2 \tau\left[\phi\left(x^{0}\right)-(1+\delta) \varepsilon\right]} d x \\
& +c \tau \int_{G}|P(x, D) v|^{2} e^{2 \tau \phi(x)} d x .
\end{aligned}
$$


Hence, where $u_{H}$ and $v_{\Omega_{\varepsilon}}$ are the restrictions of $u$ and $v$ to $H$ and $\Omega_{\varepsilon}$ respectively,

Define $a$ by

$$
\begin{aligned}
\left\|u_{H}\right\|_{(m)}^{2} \leqq & \left\|v_{\Omega_{\varepsilon}}\right\|_{(m)}^{2} \leqq c \tau e^{-2 \tau \delta \varepsilon} \int_{\Omega^{\prime} \mid \Omega_{(1+\delta) \varepsilon}}|P(x, D) v|^{2} d x \\
& +c \tau \int_{G}|P(x, D) v|^{2} e^{2 \tau\left[\phi(x)-\phi\left(x^{0}\right)+(1+\delta) \varepsilon\right]} d x .
\end{aligned}
$$

Define

$$
\sup _{x \in G} e^{2 \tau\left[\phi(x)-\phi\left(x^{0}\right)+\varepsilon\right]}=e^{2 \tau(a+\varepsilon)} .
$$

and

$$
\|u\|_{\Omega^{\prime}}^{2}=\int_{\Omega^{\prime}}|P(x, D) v|^{2} d x
$$

The estimate now reads

$$
\|u\|_{G}^{2}=\int_{G}|P(x, D) v|^{2} d x
$$

$$
\begin{aligned}
\left\|u_{H}\right\|_{(m)} & \leqq\left\|v_{\Omega_{\varepsilon}}\right\|_{(m)} \leqq c \tau^{1 / 2}\left[e^{-\tau \delta \varepsilon}\|u\|_{\Omega^{\prime}}+e^{\tau[a+\varepsilon]}\|u\|_{G}\right] \\
& \leqq c\left[e^{-\tau \varepsilon(\delta-1 / k)}\|u\|_{\Omega^{\prime}}+e^{\tau[a+(1+1 / k) \varepsilon]}\|u\|_{G}\right],
\end{aligned}
$$

for some $k>0$ such that $0<\delta-1 / k$. The constant $c$ depends on $\varepsilon, \delta, k$ and $\tau_{0}$. For $M_{0}>0$, let

$$
U_{M_{0}}=\left\{u: P(x, D) u(x)=0, x \in \Omega,\|u\|_{\Omega^{\prime}} \leqq M_{0}\right\} .
$$

Certainly, for $u \in U_{M_{0}}$,

$$
\left\|u_{H}\right\|_{(m)} \leqq\left\|v_{\Omega_{\varepsilon}}\right\|_{(m)} \leqq c\left[e^{-\tau \varepsilon(\delta-1 / k)} M_{0}+e^{\tau[a+(1+1 / k) \varepsilon]}\|u\|_{G}\right] .
$$

For $u \in U_{M_{0}}$, choose $\tau \geqq 0$ such that $\|u\|_{G}=e^{-\tau[a+(1+\delta) \varepsilon]} M_{0}$.

If $\tau \geqq \tau_{0}$, it follows that

$$
\left\|u_{H}\right\|_{(m)} \leqq\left\|v_{\Omega_{\varepsilon}}\right\|_{(m)} \leqq c M_{0}^{1-\alpha}\|u\|_{G}^{\alpha}
$$

where $\alpha=\varepsilon(\delta-1 / k)[a+(1+\delta) \varepsilon]^{-1}$.

Observe that if $0 \leqq \tau \leqq \tau_{0}$, the estimate (8) is valid with a different constant $c$. Again, it follows that

$$
\left\|u_{H}\right\|_{(m)} \leqq c M_{0}^{1-\alpha}\|u\|_{G}^{\alpha} .
$$

In exactly the same way, but with (4) used in place of $(5)$, if $P(x, D)$ is principally normal or has real coefficients, we obtain

$$
\left\|u_{H}\right\|_{(m-1)} \leqq\left\|v_{\Omega_{\varepsilon}}\right\|_{(m-1)} \leqq c \tau^{-1 / 2} M_{0}^{1-\beta}\|u\|_{G}^{\beta},
$$

for $u \in U_{M_{0}}, \tau \neq 0$. The Hölder exponent is, in this case, $\beta=\varepsilon \delta[a+(1+\delta) \varepsilon]^{-1}$.

The conclusions may be summed up in the following more formal statement.

THEOREM 1. Let $u$ satisfy $P(x, D) u(x)=0$ for $x \in \Omega$.

Under the assumptions made at the beginning of this section about the operator and about the level surface $\psi(x)=\psi\left(x^{0}\right)$, it follows that

$$
\left\|u_{H}\right\|_{(m-1)} \leqq\left\|v_{\Omega_{\varepsilon}}\right\|_{(m-1)} \leqq c \tau^{-1 / 2} M_{0}^{1-\beta}\|u\|_{G}^{\beta}
$$


when $P(x, D)$ has real coefficients or is principally normal, and

$$
\left\|u_{H}\right\|_{(m)} \leqq\left\|v_{\Omega_{\varepsilon}}\right\|_{(m)} \leqq c M_{0}^{1-\alpha}\|u\|_{G}^{\alpha}
$$

when $P(x, D)$ is elliptic, where $v=\chi u$ for $u \in C_{0}^{\infty}\left(\Omega^{\prime}\right)$ and $\chi=1$ on a neighborhood of $x^{0}$. The Hölder exponents are

$$
\beta=\varepsilon \delta[a+(1+\delta) \varepsilon]^{-1} \text { and } \alpha=\varepsilon(\delta-1 / k)[a+(1+\delta) \varepsilon]^{-1} .
$$

The constant $k$ satisfies $0<\delta-1 / k$. The positive constants $\varepsilon$ and $\delta$ are chosen suffciently small to meet requirements imposed by the geometry. The constant a is a bound for $\phi(x)-\phi\left(x^{0}\right)$ if $x$ is in $G$. As $G$ depends only on $\chi$, a depends only on $\phi$ and $\chi$. The constant $c$ depends on $\varepsilon, \delta, \tau_{0}$, and $k$. The constants $\tau_{0}$ and $k$ depend only on $P$ and $\delta$ respectively.

4. Pointwise estimates. If $u$ is the solution of an elliptic equation $P(x, D) u(x)=0$ in $\Omega$ with $m$ th order derivatives that are Hölder continuous, it is possible to convert the bound $M_{0}$ and the norms $\|u\|_{H}$ and $\|u\|_{G}$ in (9) respectively to a pointwise bound on $|u|$ in $\Omega$ and the uniform norm of $u$ in $H$ and the uniform norm of $u$ in an open set containing the closure of $G$.

Before a statement of the formal result can be given, it is necessary to observe that a version of (9) holds true for some $\varepsilon^{\prime}$ satisfying $\varepsilon^{\prime}>\varepsilon$. Thus, let

$$
G^{\prime}=\{x: 0<\chi(x)<1\} \cap \Omega_{(1+\delta) \varepsilon^{\prime}}, \quad \alpha^{\prime}=\varepsilon^{\prime}(\delta-1 / k)\left[a+(1+\delta) \varepsilon^{\prime}\right]^{-1},
$$

and let $\delta$ be the same as in Theorem 1. Choose $\varepsilon^{\prime}>\varepsilon$ such that

$$
\bar{G}^{\prime} \subset\left\{x: \psi\left(x^{0}\right)<\psi(x)\right\} .
$$

This choice of $\varepsilon^{\prime}$ is possible since the set $\left\{x: \psi\left(x^{0}\right)<\psi(x)\right\}$ contains the closure of

$$
G=\{x: 0<\chi(x)<1\} \cap \Omega_{(1+\delta) \varepsilon} .
$$

The derivation in $\S 4$ may be repeated with $\varepsilon^{\prime}, \alpha^{\prime}$, and $G^{\prime}$ in place of $\varepsilon, \alpha$ and $G$ respectively. The result is that

$$
\left\|u_{H}\right\|_{(m)} \leqq\left\|v_{\Omega_{\varepsilon^{\prime}}}\right\|_{(m)} \leqq c M_{0}^{1-\alpha^{\prime}}\|u\|_{G^{\prime}}^{\alpha}
$$

Let $N$ be a neighborhood of $x^{0}$ in $\left\{x: \psi\left(x^{0}\right)<\psi(x)\right\}$. Observe that the constant $\varepsilon^{\prime}$ may also be chosen such that if $\bar{G}$ is contained in the interior of $N$, then $\bar{G}^{\prime}$ is contained in the interior of $N$ as well.

Theorem 2. Let $N, \varepsilon^{\prime}$ and $G^{\prime}$ satisfy the definitions and properties in the paragraphs just above. Let $P(x, D)$ be an elliptic operator for which $(9)$ is valid and let $u$ satisfy $P(x, D) u(x)=0$ in $\Omega$. Assume $u$ and the derivatives of $u$ up to order $m$ are defined and continuous on the closure of $\Omega$. Also assume that the mth order derivatives of $u$ are Hölder continuous in $\bar{\Omega}$ with, say, exponent $\beta$. Let $M$ be an upper bound for $|u(x)|$ for $x \in \bar{\Omega}$. Let $\xi \in \bar{H}$. Let $B(\xi, R)$ denote the ball of radius $R$ with center at $\xi$. Choose $R$ such that $B(\xi, R) \subset \Omega_{\varepsilon^{\prime}}$. For $\alpha^{\prime}$ defined in (10), it follows that: 
(i) If $n / 2<m$, then

$$
|u(\xi)| \leqq c R^{-n} M^{1-\alpha^{\prime}}\left\|u_{N}\right\|_{\infty}^{\alpha^{\prime}} ;
$$

(ii) if $2 \leqq m \leqq n / 2$ and $3<n$, then

$$
|u(\xi)| \leqq c \nu R^{-\mu} M^{s / p}\left[M^{1-\alpha^{\prime}}\left\|u_{N}\right\|_{\infty}^{\alpha^{\prime}}\right]^{1 / p}
$$

where $1 / p+1 / q=1, s=1 /(q-1), \mu=n-n / q, \nu=[q(m-n)+n]^{-1 / q}$ and $q$ is chosen such that $q(m-n)+n>0$. The constant c depends on $m, \Omega, \Omega^{\prime}, N, G, \varepsilon^{\prime}, \delta$, the ellipticity constant of $P$ and bounds for the $L_{\infty}(\Omega)$ norms of the coefficients of $P$.

Proof. Let $u$ satisfy $P(x, D) u(x)=0$ in $\Omega$. Recall that $M_{0}$ is an upper bound for $\|u\|_{\Omega^{\prime}}$, that is,

$$
\|u\|_{\Omega^{\prime}}^{2}=\int_{\Omega^{\prime}}|P(x, D) v|^{2} d x \leqq M_{0}^{2} .
$$

By taking the maximum on $\Omega^{\prime}$ of the quantities inside the integral, it is clear that

$$
\|u\|_{\Omega^{\prime}}^{2} \leqq \text { const } \sum_{|\rho|=0}^{m-1} \sup _{x \in \Omega^{\prime}}\left|D^{\rho} u(x)\right|^{2} .
$$

Recall that $\bar{\Omega}^{\prime} \subset \Omega$. Since the $m$ th order derivatives of $u$ are Hölder continuous, Schauder type interior estimates (see [3, p. 232]) can be applied to show that the sum

$$
\sum_{|\rho|=0}^{m-1} \sup _{x \in \Omega^{\prime}}\left|D^{o} u(x)\right|
$$

is bounded by a constant, $c$, times the maximum of $|u|$ on $\bar{\Omega}$ and hence by $c M$. Thus,

$$
\|u\|_{\Omega^{\prime}}^{2} \leqq \text { const } \sum_{|\rho|=0}^{m-1} \sup _{x \in \Omega^{\prime}}\left|D^{\rho} u(x)\right|^{2} \leqq \text { const } M^{2} .
$$

It follows that the global bound $M_{0}$ in (11) may be replaced by the pointwise bound $M$ for $|u(x)|, x \in \Omega$.

The interior estimates also yield a uniform bound for

$$
\|u\|_{G^{\prime}}^{2}=\int_{G^{\prime}}|P(x, D) v(x)|^{2} d x .
$$

Thus, $\|u\|_{G^{\prime}} \leqq$ const $\left\|u_{N}\right\|_{\infty}$. Hence,

$$
\left\|u_{H}\right\|_{(m)} \leqq\left\|v_{\Omega_{\varepsilon^{\prime}}}\right\|_{(m)} \leqq c M^{1-\alpha^{\prime}}\left\|u_{N}\right\|_{\infty}^{\alpha^{\prime}}
$$

where the constant $c$ depends on $m, \Omega, \Omega^{\prime}, N, G^{\prime}$, the ellipticity constant of $P(x, D)$ and bounds for the $L_{\infty}(\bar{\Omega})$ norms of the coefficients of $P(x, D)$.

It remains to replace the Sobolev norm $\left\|u_{H}\right\|_{(m)}$ by the uniform norm $\left\|u_{H}\right\|_{\infty}$. Let $\xi \in \bar{H}$. Choose $R$ such that $B(\xi, R) \subset \Omega_{\varepsilon^{\prime}}$. There are two cases according as whether $n / 2<m$ or whether $(2 \leqq) m \leqq n / 2$. 
If $n / 2<m$, then by the lemma of Sobolev [9, p. 282], it follows that

$$
|u(\xi)|=|v(\xi)| \leqq c R^{-n}\left\|v_{\Omega_{\varepsilon^{\prime}}}\right\|_{(m)}
$$

where $c$ depends only on $n$. Observe that $n / 2<m$ when $n \leqq 3$, which is the case of greatest physical significance.

If $m \leqq n / 2$, a pointwise estimate can be derived under the assumption that $|u|$ is bounded on $\Omega$. The derivation begins with the remark that in one proof of a version of the Sobolev lemma $[9$, p. 282], the following identity is established:

$$
u(\xi) \int_{\sigma} d \sigma=(-1)^{m} /(m-1) ! \int_{\sigma} \int_{0}^{R} r^{m-1} \frac{\partial^{m}}{\partial r^{m}}(h u) d r d \sigma
$$

where $r=|x-\xi|, \sigma$ is the $(n-1)$-dimensional unit sphere, $h(t)=g(t / R)$ and $g$ is a $C^{\infty}$ function such that $g(t)=1$ for $t \leqq \frac{1}{2}$ and $g(t)=0$ for $1 \leqq t$. Hölder's inequality implies that

$$
|u(\xi)| \leqq \text { const }\left[\int_{B(\xi, R)}\left|\frac{\partial^{m}}{\partial r^{m}}(h u)\right|^{p} d x\right]^{1 / p}\left[\int_{\sigma} \int_{0}^{R} r^{(m-n) a} r^{n-1} d r d \sigma\right]^{1 / q},
$$

where $1 / p+1 / q=1, p$ and $q$ positive.

Recall that $3<n$. Since $2 \leqq m \leqq n / 2$, it follows that $n / 2 \leqq n-m \leqq n-2$. The second integral above on the right is bounded if $(m-n) q+n>0$. This condition is satisfied by the proper choice of $q$ such that $1<q<2$. In fact, $q<n /(n-m)$ and $n /(n-2)$ $\leqq n /(n-m) \leqq 2$. Hence,

$$
\int_{\sigma} \int_{0}^{R} r^{(m-n) q} r^{n-1} d r d \sigma=R^{q(m-n)+n}[q(m-n)+n]^{-1} \int_{\sigma} d \sigma .
$$

Schwarz's inequality shows that the first integral of (13) is bounded by

$$
\int_{B(\xi, R)}\left|\frac{\partial^{m}}{\partial r^{m}}(h u)\right|^{2} d x \int_{B(\xi, R)}\left|\frac{\partial^{m}(h u)}{\partial r^{m}}\right|^{2 s} d x
$$

where $s=p-1=1 /(q-1)$. Since $\left|d^{k} h(t) / d t^{k}\right| \leqq A_{k} / R^{k}$, Leibniz's rule implies that

$$
\left|\frac{\partial^{m}}{\partial r^{m}}(h u)\right| \leqq \text { const } R^{-m} \sum_{|\rho| \leqq m}\left|\frac{\partial^{\rho} u}{\partial x^{\rho}}\right| .
$$

Therefore,

and

$$
\begin{aligned}
\int_{B(\xi, R)}\left|\frac{\partial^{m}}{\partial r^{m}}(h u)\right|^{2} d x & \leqq \text { const } R^{-2 m} \sum_{|\rho| \leqq m} \int_{B(\xi, R)}\left|\frac{\partial^{\rho} u}{\partial x^{\rho}}\right|^{2} d x \\
& \leqq \text { const } R^{-2 m}\left\|u_{H}\right\|_{(m)}^{2}
\end{aligned}
$$

$$
\left|\frac{\partial^{m}}{\partial r^{m}}(h u)\right|^{2 s} \leqq \text { const } R^{-2 m s}\left[\sum_{|\rho| \leqq m}\left|\frac{\partial^{\rho} u}{\partial x^{\rho}}\right|\right]^{2 s} .
$$

If $M$ is the bound on $|u|$ in $\Omega$, given in the hypothesis, then, by the interior estimates 
[3, p. 232], a constant multiple of $M$ is a bound on the derivatives of $u$ up to order $m$ in $B(\xi, R)$. Hence

$$
\left|\partial^{m}(h u) / \partial r^{m}\right|^{2 s} \leqq \text { const } M^{2 s} R^{-2 s m}
$$

It follows that

$$
\left[\int_{B(\xi, R)}\left|\frac{\partial^{m}}{\partial r^{m}}(h u)\right|^{p} d x\right]^{2} \leqq \text { const } R^{-2 m}\left\|u_{H}\right\|_{(m)}^{2} M^{2 s} R^{-2 s m}
$$

Hence, (13) becomes

$$
\begin{aligned}
|u(\xi)| & \leqq\left[R^{-m}\left\|u_{H}\right\|_{(m)} R^{-s m} M^{s}\right]^{1 / p}\left[R^{q(m-n)+n} / q(m-n)+n\right]^{1 / q} \\
& =M^{s / p} \nu R^{-\mu}\left[\left\|u_{H}\right\|_{(m)}\right]^{1 / p} \leqq M^{s / p} \nu R^{-\mu}\left[\left\|v_{\Omega_{\varepsilon}}\right\|_{(m)}\right]^{1 / p}
\end{aligned}
$$

where $\mu=n-n / q$ and $\nu=[q(m-n)+n]^{-1 / q}$. The estimate in (ii) now follows from (12).

5. Pseudo-convexity. In order to obtain Hölder continuous dependence across a surface through a point $x^{0}$ for the continuation of a solution of an elliptic equation, it is sufficient to know there exists a real-valued function $\psi \in C^{2}(\Omega)$ such that $\nabla \psi\left(x^{0}\right) \neq 0$ and the surface defined by $\psi(x)=\psi\left(x^{0}\right)$ is strongly pseudo-convex at $x^{0}$ with respect to the operator. The existence of $\psi$ is simplified for some second order elliptic operators. The operators discussed here for which this simplification is possible are those with real coefficients and those that can be factored. In fact for the class of second order operators with real $C^{1}$ coefficients, the existence of $\psi$ is a complete triviality: For, suppose we have an operator with real coefficients $a_{j k} \in C^{1}(\Omega)$ that is elliptic in $\Omega$. Let $\zeta=\xi+i \tau \nabla \psi(x)$, where $\psi$ is any $C^{2}$ function for which $\nabla \psi(x) \neq 0$. The condition (3) in the definition of strong pseudo-convexity, namely,

$$
P_{m}(\zeta)=0 \text { and } \sum_{j} P_{m}^{(j)}(x, \zeta) \frac{\partial \psi}{\partial x_{j}}=0,
$$

takes the form $P_{m}(\zeta)=0$ and

$$
\sum_{j, k} a_{j k}(x)\left(\xi_{k}+i \tau \frac{\partial \psi}{\partial x_{k}}(x)\right) \frac{\partial \psi}{\partial x_{j}}(x)=0 .
$$

Therefore,

$$
0=\sum_{j, k} a_{j k}(x) \frac{\partial \psi}{\partial x_{k}}(x) \frac{\partial \psi}{\partial x_{j}}(x) \geqq \alpha|\nabla \psi(x)|^{2}>0,
$$

where $\alpha$ is the ellipticity constant. Hence, there is no $\zeta$ for which (2) must be verified, and $\psi$ meets the requirements of the second half of the definition. The first half is satisfied even more trivially since the operator is elliptic. This completes the case for $P$ with real coefficients.

The assumption that the polynomial $P(x, \zeta)$ can be factored at $x$ considerably simplifies the computations that are necessary to make in order to verify strong 
pseudo-convexity. This is the other kind of second order operator we discuss. Thus, suppose

$$
P(x, \zeta)=P(\zeta)=\left(\sum_{j} a_{j} \zeta_{j}\right)\left(\sum_{j} b_{j} \zeta_{j}\right) .
$$

If $P$ is elliptic, only (2) must be verified when (3) is fulfilled. Hence, assume, $P(\zeta)=0$, and

where $\zeta=\xi+i \tau \nabla \psi(x)$.

$$
\sum_{j} P^{(j)}(\zeta) \frac{\partial \psi}{\partial x_{j}}(x)=0
$$

First, observe that $P^{(j)}(\zeta) \neq 0$ for some $j$ is a necessary condition that a strongly pseudo-convex function $\psi$ must satisfy. For, if $\psi$ is strongly pseudo-convex, and if $\phi=e^{\lambda \psi}$ for $\lambda$ sufficiently large, then, by [13, Theorem 8.6 .3$]$,

$$
\sum_{|\alpha| \leqq m} \tau^{2(m-|\alpha|)} \int\left|D^{\alpha} u\right|^{2} e^{2 \tau \phi} d x \leqq K \tau \int|P(x, D) u|^{2} e^{2 \tau \phi} d x,
$$

$u \in C_{0}^{\infty}\left(\Omega^{\prime}\right)$, for some neighborhood $\Omega^{\prime}$ of $x$. For this estimate to hold, it is necessary $[13$, Theorem 8.1.1] that

$$
|\zeta|^{2(m-1)} \leqq 2 K_{1}\left\{\sum_{j, k} \frac{\partial^{2} \phi}{\partial x_{j} \partial x_{k}} P^{(j)}(x, \zeta) \overline{P^{(k)}(x, \zeta)}+\tau^{-1} \operatorname{Im} \sum_{k} P_{k}\left(x, \zeta \overline{P^{(k)}(x, \zeta)}\right\}\right.
$$

for $\zeta=\xi+i \sigma \nabla \phi(x)$, with $\xi \in R_{n}$ and $0 \neq \sigma \in R_{1}$ satisfying $P(x, \zeta)=0$. Since $|\zeta| \neq 0$ and $\nabla \psi(x)=c_{x} \nabla \phi(x)$, the estimate cannot hold if $P^{(k)}(x, \xi+i \tau \nabla \psi(x))=0$ for each $k$.

The fact that $P^{(k)}(\zeta) \neq 0$ for some $k$ implies only one of the factors, say $\sum_{j} b_{j} \zeta_{j}$, of $P(\zeta)=0$ can be zero. For,

$$
P^{(k)}(\zeta)=a_{k} \sum_{j} b_{j} \zeta_{j}+b_{k} \sum_{j} a_{j} \zeta_{j}=b_{k} \sum_{j} a_{j} \zeta_{j} \neq 0
$$

implies $\sum_{j} a_{j} \zeta_{j} \neq 0$.

The condition $\sum_{j} P_{m}^{(j)}(\zeta) \partial \psi(x) / \partial x_{j}=0$ now yields

But,

$$
\begin{array}{rl}
0=2 & P(\zeta)=\sum_{j} P^{(j)}(\zeta) \zeta_{j}=\sum_{j} P^{(j)}(\zeta) \xi_{j} \\
& +\sum_{j} P^{(j)}(\zeta) i \tau \frac{\partial \psi}{\partial x_{j}}(x)=\sum_{j} P^{(j)}(\zeta) \xi_{j}=0 .
\end{array}
$$

$$
\begin{aligned}
0=\sum_{j} P^{(j)}(\zeta) \xi_{j} & =\sum_{j}\left(a_{1} \zeta_{1}+\cdots+a_{n} \zeta_{n}\right) b_{j} \xi_{j} \\
& =\left(\sum_{j} a_{j} \zeta_{j}\right)\left(\sum_{k} b_{k} \xi_{k}\right)=0 .
\end{aligned}
$$

Therefore, $\sum_{j} b_{j} \xi_{j}=0$ since $\sum_{j} a_{j} \zeta_{j} \neq 0$. Therefore,

$$
P(\xi)=\left(\sum_{j} a_{j} \xi_{j}\right)\left(\sum_{k} b_{k} \xi_{k}\right)=0,
$$

which is impossible as $P$ is elliptic and $\xi$ is real, unless $\xi=0$. 
Hence, it remains to verify that (2) can hold when $\xi=0$. When it is written out for the case

$$
P(z)=\left(\sum_{j} a_{j} z_{j}\right)\left(\sum_{k} b_{k} z_{k}\right)
$$

with $\sum_{j} b_{j} \zeta_{j}=0$ and $P^{(j)}(\zeta)=b_{j} \sum_{k} a_{k} \zeta_{k}$, verification of condition (2) reduces to showing that

$$
\left|\sum_{j} a_{j} \zeta_{j}\right|^{2} \sum_{j, k} \psi_{x_{j} x_{k}} b_{j} \bar{b}_{k}+\tau^{-1} \operatorname{Im}\left|\sum_{j} a_{j} \zeta_{j}\right|^{2} \sum_{k}\left(\sum_{j} \frac{\partial b_{j}}{\partial x_{k}} \zeta_{j}\right) \bar{b}_{k}>0 .
$$

Let $b_{j}=\beta_{j}+i \gamma_{j}$ and $\eta_{j}=\partial \psi / \partial x_{j}(x)$. It follows, since $\xi=0$, that

$$
\tau^{-1} \operatorname{Im} \sum_{k}\left(\sum_{j} \frac{\partial b_{j}}{\partial x_{k}} \zeta_{j}\right) \tilde{b}_{k}=\sum_{j, k}\left[\eta_{j} \gamma_{k} \frac{\partial \gamma_{j}}{\partial x_{k}}+\eta_{j} \beta_{k} \frac{\partial \beta_{j}}{\partial x_{k}}\right] .
$$

Therefore, (2) reduces to

$$
\left|\sum_{j} a_{j} \zeta_{j}\right|^{2}\left[\sum_{j, k} \frac{\partial^{2} \psi}{\partial x_{j} \partial x_{k}} b_{j} \bar{b}_{k}+\sum_{j, k}\left(\eta_{j} \gamma_{k} \frac{\partial \gamma_{j}}{\partial x_{k}}+\eta_{j} \beta_{k} \frac{\partial \beta_{j}}{\partial x_{k}}\right)\right] .
$$

All quantities are fixed in the second sum in the brackets. Hence, the term is positive whenever the eigenvalues of $\left(\partial^{2} \psi(x) / \partial x_{i} \partial x_{k}\right)$ are sufficiently large, since $\left|\sum_{j} a_{j} \zeta_{j}\right| \neq 0$ and since $\left(b_{1}, \ldots, b_{n}\right) \neq 0$.

\section{REFERENCES}

1. N. Aronszajn, $A$ unique continuation theorem for solutions of elliptic partial differential equations or inequalities of second order, J. Math. Pures Appl. 36 (1957), 235-249.

2. L. Bers and L. Nirenberg, On a representation theorem for linear elliptic systems with discontinuous coefficients and its applications, Atti del convegno internazionale sulle equazione alle derivate parzialli, Trieste, 1954, pp. 111-140.

3. L. Bers, F. John and M. Schecter, Partial differential equations, Interscience, New York, 1964.

4. T. Carleman, Sur les systèmes linéaires aux dérivées partielles du premier ordre a deux variables independentes, C. R. Acad. Sci. Paris 197 (1933), 471-474.

5. H. Cordes, Uber die eindeutige Bestimmtheit der Lösungen elliptischer Differentialgleichungen durch Anfangsvorgaben, Nachr. Akad. Wiss. Göttingen Math.-Phys. Kl. IIa (1956), 239-258.

6. J. Douglas, Jr., "Approximate continuation of harmonic and parabolic functions," in Numerical solution of partial differential equations, J. H. Bramble (editor), Academic Press, New York, 1966.

7. - "A numerical method for analytic continuation," in Boundary problems in differential equations, R. E. Langer (editor), Univ. of Wisconsin Press, Madison, 1960.

8. A. Douglis, Uniqueness in Cauchy problems for elliptic systems of equations, Comm. Pure Appl. Math. 6 (1953), 291-298; 13 (1960), 593-607.

9. A. Friedman, Partial differential equations of parabolic type, Prentice-Hall, Englewood Cliffs, N. J., 1964. 
10. Ju. K. Gerasimov, The three-sphere theorem for a class of elliptic equations of high order and a refinement of this theorem for a linear elliptic equation of second order, Amer. Math. Soc. Transl. 72 (1968), 135-162.

11. E. Heinz, Uber die Eindeutigkeit beim Cauchyschen Anfangswertproblem eins elliptischen Differentialgleichung zweiter Ordnung, Nachr. Akad. Wiss. Göttingen Math.-Phys. K1. IIa (1955), 1-12.

12. E. Hille, Analytic function theory, Vol. II, Ginn, Boston, 1962, p. 410.

13. L. Hörmander, Linear partial differential operators, Academic Press, New York, 1963.

14. F. John, Continuous dependence on data for solutions of partial differential equations with a prescribed bound, Comm. Pure Appl. Math. 13 (1960), 551-585.

15. E. Landis, A three-spheres theorem, Soviet Math. Dokl. 4 (1963), 76-78.

16. $\mathrm{K}$. Miller, Three circle theorems in partial differential equations and their applications to improperly posed problems, Arch. Rational Mech. Anal. 16 (1964), 126-154.

17. C. Morrey, Jr., On the analyticity of solutions of analytic non-linear elliptic systems of partial differential equations, Amer. J. Math. 80 (1958), 198-237.

18. C. Müller, On the behavior of the solutions of the differential equation $\Delta u=F(x, u)$ in the neighborhood of a point, Comm. Pure Appl. Math. 7 (1954), 505-551.

19. M. Protter, Unique continuation for elliptic equations, Trans. Amer. Math. Soc. 95 (1960), 81-91.

UNIVERSITY OF ILLINOIS,

URBANA, ILLINOIS 